\title{
Pervasive blood pressure monitoring using Photoplethysmogram (PPG) Sensor
}

\author{
Farhan Riaz ${ }^{\mathrm{a}}$, Muhammad Ajmal Azad ${ }^{\mathrm{b}}$, Junaid Arshad ${ }^{\mathrm{c}}$, Muhammad Imran ${ }^{\mathrm{d}}$, Ali Hassan ${ }^{\mathrm{a}}$, Saad Rehman \\ ${ }^{a}$ Department of Computer and Software Engineering, National University of Science and Technology, Islamabad, Pakistan \\ ${ }^{b}$ Department of Computer Science and Mathematics, The University of Derby, Derby, United Kingdom \\ ${ }^{c}$ School of Computing and Engineering, University of West London, London, United Kingdom \\ ${ }^{d}$ College of Applied Computer Science, King Saud University, Saudi Arabia
}

\begin{abstract}
Preventive healthcare requires continuous monitoring of the blood pressure (BP) of patients, which is not feasible using conventional methods. Photoplethysmogram (PPG) signals can be effectively used for this purpose as there is a physiological relation between the pulse width and BP and can be easily acquired using a wearable PPG sensor. However, developing real-time algorithms for wearable technology is a significant challenge due to various conflicting requirements such as high accuracy, computationally constrained devices, and limited power supply. In this paper, we propose a novel feature set for continuous, real-time identification of abnormal BP. This feature set is obtained by identifying the peaks and valleys in a PPG signal (using a peak detection algorithm), followed by the calculation of rising time, falling time and peak-to-peak distance. The histograms of these times are calculated to form a feature set that can be used for classification of PPG signals into one of the two classes: normal or abnormal BP. No public dataset is available for such study and therefore a prototype is developed to collect PPG signals alongside BP measurements. The proposed feature set shows very good performance with an overall accuracy of approximately $95 \%$. Although the proposed feature set is effective, the significance of individual features varies greatly (validated using significance testing) which led us to perform weighted voting of features for classification by performing autoregressive modeling. Our experiments show that the simplest linear classifiers produce very good results indicating the strength of the proposed feature set. The weighted voting improves the results significantly, producing an overall accuracy of about $98 \%$. Conclusively, the PPG signals can be effectively used to identify BP, and the proposed feature set is efficient and computationally feasible for implementation on standalone devices.
\end{abstract}

Keywords: Butterworth filters, Averaging filters, Classification, Photoplethysmogram (PPG).

\section{Introduction}

Wearable health monitoring technologies (smart watches, fitness tracker, smart wristbands) are becoming widely used by consumers for tracking and monitoring their health conditions. It is estimated that more than 240 million wearable devices will be shipped annually by the year 2021 [1] with an estimated revenue to cross US\$ 30 Billion. Today, modern wearable devices can perform sophisticated tasks and constantly monitor health indicators, such as glucose measurements in the blood, heart rate variability, blood pressure readings, calories burned, steps walked etc. Heart rate variability (HRV) is the most important parameter for predicting major cardiovascular diseases in both the general population and patients. Nowadays, non-invasive detective methods have become a basic requirement for the wearable medical devices, especially in the scenario, when devices are used for the personal health monitoring without assistance from the nursing staff $[2,3,4]$. The bio-signal is highly related to the cardiovascular conditions and thus can be an efficient way to detect the heart diseases by estimating the Blood Pressure $(B P)$.
One way to measure the $\mathrm{BP}$ is through arterial tonometer $[5,6]$. The heart-to-beat waveform can be measured from arterial tonometer but is strongly subject to motion artifacts caused by high sensitivity, sensor placement and movement [7]. Cuff sphygmomanometer is the most standard non-invasive technique that is used to measure $\mathrm{BP}$ [8]. Continuous measurement of BP using this technique requires periodic cuff inflation and deflation, thus is not suitable for the continuous measurement of BP. The correlation between BP and Pulse Transit Time (PTT) has also been used to estimate the BP $[9,10,11]$. Specifically, PTT is the time interval for the arterial pulse pressure wave to travel from the aortic valve to the peripheral site. It is widely known that the PTT varies inversely with BP changes and can be used for the cuffless and continuous estimation of BP. Usually, the time interval between R-wave in the electrocardiography (ECG) signal and the peak of the peripheral pulse is selected as the PTT [12]. The peripheral pulses are recorded at the fingertips using PPG signals sensors (Fig. 1).

The risk of having hypertension (BP issues) is no longer imminent only in aged patients but is also the younger peo- 
ple are also having the risk of cardiovascular diseases [13]. To identify cardiovascular events, the Electrocardiogram signal has been widely used for tracking abnormalities in patients. However, to collect the ECG parameters, it is necessary that several bio-electrodes are placed at various parts of the human body which might become complex in emergency conditions and also requires the assistance of technical personnel. Alternatively, Photoplethysmography (PPG) can be used as a heart monitoring technique. Specifically, PPG technique uses infrared light to measure the blood volume changes in the microvascular bed of tissues by using a pulse oximeter [14].

To effectively calculate BP, most of the previous works are focused on the calculation of PTT from both the PPG and the ECG signals. This requires affixing of sensors on the chest of the patients due to which, the calculation of $\mathrm{BP}$ on the fly is not feasible for the continuous seamless monitoring. More recently, researchers have found that PPG signals can be used for estimating BP levels. This presents two major gaps in this area of research: 1) Given that the aim of this work is to use wearable sensors for preventive healthcare, it is imperative that the algorithms developed can work in real-time on devices which are computationally less powerful, and 2) The system should be able to raise alarms for commercial usage of the devices in a more reliable manner. In this context, it is possible to further simplify the research problem: since the work is mainly focused on preventive healthcare [15], the aim of systems is more inclined towards the detection of abnormal BP rather than being able to measure the exact value of BP as already achieved in [16]. In this paper, we aim to propose a novel method to detect an abnormality in the BP using PPG signals acquired from the pulse oximeter sensor. The method is based on de-noising of the signal, followed by the peak detection in PPG signals. After peak detection, different features are extracted which are then passed through the machine learning module for detecting abnormalities in BP. The major contributions of this paper are as follows.

- Data collection: We have devised an experimental protocol that can be used to collect PPG dataset. The proposed system uses an embedded system (Arduino board) and the pulse sensor to collect the data. Along with the PPG signals, the BP readings of the subjects are simultaneously recorded to validate the correlation between the physiology of PPG signals and BP.

- Feature extraction: We have proposed a novel temporal feature set that can be used for the identification of BP. Prior to feature extraction, de-noising of the signals is performed for detecting the peaks and valleys in a PPG signal. This is followed by the features that have been proposed i.e. the rising time, falling time and peak-to-peak distance.

- Feature analysis: We analyzed the statistical signif-

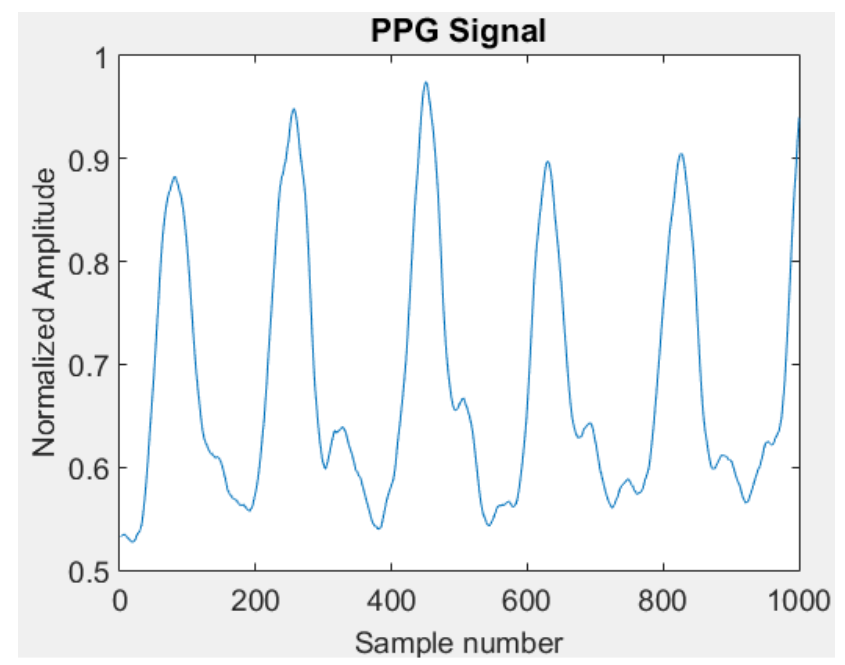

Figure 1: A PPG signal obtained after preprocessing followed by butterworth filtering.

icance of each feature set by calculating their variations for the normal and abnormal cases and calculate their individual and collective performances using different machine learning methods.

- Weighted voting: We have proposed a feature voting strategy based on autoregressive modeling to calculate weights assigned to individual features in the least square manner which significantly improves the classification results.

Rest of the paper is organized as follows: We review existing work (Section 2) followed by a description of dataset (Section 3) . Later, we present the proposed approach (Section 4) followed by a description of the experimental setup (Section 5). The classification results are presented in Section 6 and detailed discussion is provided in Section 7 .

\section{Background and State of the Art}

This paper is focused on using PPG signals to achieve continuous measurement of BP for elderly and vulnerable patients by exploiting the physiological relation between pulse width and BP. In particular, we model this challenge as a general classification problem leveraging features which can be used to identify if a patient has a normal or abnormal BP. Within this context, the research presented in this paper is relevant to multiple domains such as analysis of PPG signals and classification. In this section, we present background on the PPG signal, the motivation of this work and state of the art on the use of estimating the blood pressure through sensor devices.

\subsection{Photoplethysmogram (PPG)}

$\mathrm{PPG}$ is a pulsating wave produced by a pulse oximeter and has been traditionally used to measure oxygen saturation, blood pressure, and other vital functions. A pulse 
oximeter is often used to have a PPG which measures light changing and absorption through the skin. With each cardiac cycle, the heart pumps blood to the periphery [17],[18]. Even though this pressure pulse is somewhat damped by the time it reaches the skin, it is enough to distend the arteries and arterioles in the subcutaneous tissue. The change in volume caused by the pressure pulse is detected by illuminating the skin with the light from a lightemitting diode (LED) and then measuring the amount of light either transmitted or reflected a photodiode (Fig. 2). Because blood flow to the skin can be modulated by multiple other physiological systems, the PPG can also be used to monitor breathing, hypovolemia, and other circulatory conditions [19]. Figure 1 represents the PPG signal taken at the fingertip. It shows the movement of blood in the form of wavelike motion in the vessels, which start from the heart and ends at the fingertips. Each cardiac cycle starts with the peak signal. Beside fingertips, PPG signal can also be obtained from other body parts, for example, the vagina (vaginal photoplethysmograph), the clitoris (clitoral photoplethysmograph) and the esophagus.

\subsection{Motivation}

The blood pressure and heart rate monitoring approaches based on the PPG sensors or wearable have many advantages over the ECG-based monitoring systems. For example, the PPG device needs to be placed at a single part of the body thus minimizing the overall cost. On the other hand, the traditional ECG based approach requires to place the ECG bioelectrodes to be placed at least three places(such as the left arm, right arm, and right leg). The PPG device is easy to use and can be used even under serious conditions. Lately, PPG signals have been widely used for analysis purposes. For instance, Fleming et al. [21]

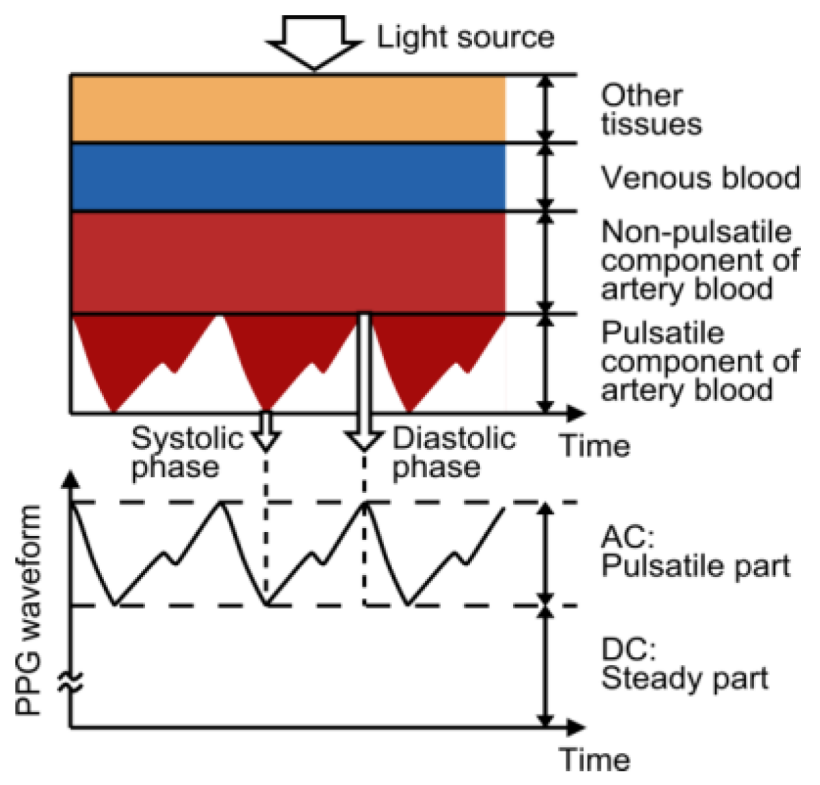

Figure 2: Principle of the PPG signals (adapted from [20]). present a comparative analysis of different signal processing techniques to extract breathing rate from the PPG.In addition to this, PPG signal has been also been used to extract the breathing rate [22] [23] [24]. Whether used to monitor breathing rate or blood pressure, a common noninvasive technique to gather PPG signal is through the fingertip of the patient. In this context, Elgendi [25] provides an account of challenges in the pre-processing stage which can influence the quality of the PPG signal and therefore make any diagnosis based on it. These challenges include powerline interference, motion artifact, a low amplitude PPG signal, and premature ventricular contraction.

\subsection{State of the Art}

One of the motivations of using PPG for blood pressure monitoring is to achieve a continuous and non-invasive way of conducting the measurements. Contrary to the traditional method of using cuff-based sphygmomanometer, there have been advancements in wearable technologies promoting non-invasive measurements. These are termed as cuffless, and systems such as Finapres (Finapres Medical Systems, Netherlands) represent such systems. Sharma et al. [26] present a review of recent developments in blood pressure monitoring using cuffless approaches, highlighting challenges such as calibration frequency and the inclusion of heart rate as part of measurements to improve the performance efficiency. Su et al. [27] presented a design that estimates the systolic blood pressure of patient or object through the use of non-wearable hydraulic bed sensor system that is placed underneath the mattress of a patient. The design requires the bed sensors and only achieve an accuracy of a maximum of $90 \%$. With respect to the use of PPG for blood pressure monitoring, Shaltis et al. [28] present efforts to develop a self-contained, wearable blood pressure monitoring sensor using PPG. Authors have focused on the miniaturization of the device so as to address the physical hindrance caused by the traditional cuff monitors when operated for continuous real-time monitoring. In fact, this approach is not isolated and there are multiple different efforts to creating devices and methods to achieve no-intrusive blood pressure monitoring using PPG as illustrated in $[24,29,30,31,32]$. A common characteristic shared by these approaches is their focus on creating a smaller cuff (for instance a finger cuff) envisaged to reduce the hindrance caused due to traditional sphygmomanometer. In addition to these approaches, [33, 34, 35] represent efforts to introduce novel methods to achieve continuous PPG measurements for blood pressure monitoring such as on a toilet seat which can be beneficial for elderly and less mobile patients. In addition to the above efforts, Kurylyak et al. [36] proposed a neural networks based approach to estimate blood pressure from the PPG signal. The authors used 21 different parameters to define input vectors for the heartbeats which have been then analyzed conducting a comparative performance analysis between neural networks and linear regression models. Ahmed et al. [37] proposed cloud-based automated heart monitoring 
system. To this extent, the authors collected data from subjects using the Electrocardiogram (ECG) wearable devices that measure the heart rates of the subject. The system provides on-demand and urgent monitoring to people living in the remote areas of the country. Rodrigues et al. [38] present a comprehensive review of systems that have proposed for healthcare systems based on the Internet of Health Things (IoHT). Authors have identified challenges in the design of IoHT system and provided a system for future trends. Mukhtar et al. [39] analyzed the cloud of Things (CoT) architectures from the perspective of its usage for efficient and smart healthcare.

: Recently, researchers have opted to perform feature extraction from PPG signals using temporal, spectral and hybrid (wavelet based) features [40, 41, 42] but all these methods pose certain limitations: the temporal-based methods are generally using some features that are based on individual samples from the signals such as maxima, minima etc. Such features are not statistical and may actually be the results of some outliers such as the displacement of the sensors, movement artifacts etc. The spectral features are generally more useful as they can help in the reduction of noise but it should be noted that the feature extraction technique should be computationally less expensive. In this paper, we address these issues by using temporal features which take the statistical properties of the PPG signals into consideration, thus proving to be a powerful feature set for the detection of abnormal BP from PPG signals.

Classification is a well-establish research domain within mathematics and statistical analysis with one of the early works presented by Fisher in [43]. In modern scientific developments, classification is generally understood coupled with machine learning approaches such as decision trees, support vector machines, neural networks, and genetic algorithms. As classification approaches generally aim to identify patterns or feature representations to segregate items within a dataset, its applications are wideranging such as customer churn analysis [44], intrusion detection [45, 46], healthcare monitoring [47] and spam call identification[48]. All these approaches aim to identify a set of features which can facilitate segregating individual data items followed by the application of supervised or unsupervised machine learning algorithms to achieve an intelligent and efficient classification of data under consideration.

\section{Dataset}

The need to acquire this dataset arises from the fact that until now, to the best of our knowledge, there is no data collected from the PPG sensor for which the BP readings are also available. The main challenge in collecting such data is that the most commonly used PPG finger sensors used in the emergency departments of the hospitals are typically restricted for access. Secondly, any intervention with the hospital equipment is typically not allowed
Table 1: Details of the collected data.

\begin{tabular}{lllll}
\hline Name & Gender & Age & BP & Class \\
\hline Subj1 & M & 20 & $110 / 70$ & Normal \\
Subj2 & M & 21 & $110 / 90$ & Normal \\
Subj3 & F & 20 & $110 / 80$ & Normal \\
Subj4 & F & 21 & $100 / 70$ & Normal \\
Subj5 & F & 22 & $100 / 60$ & Normal \\
Subj6 & M & 23 & $120 / 80$ & Normal \\
Subj7 & M & 21 & $120 / 80$ & Normal \\
Subj8 & F & 42 & $140 / 70$ & High \\
Subj9 & F & 55 & $170 / 90$ & High \\
Subj10 & F & 50 & $150 / 90$ & High \\
Subj11 & F & 60 & $150 / 90$ & High \\
Subj12 & F & 60 & $140 / 80$ & High \\
Subj13 & F & 55 & $150 / 100$ & High \\
Subj14 & F & 37 & $90 / 60$ & Low \\
Subj15 & F & 19 & $90 / 60$ & Low \\
Subj16 & F & 40 & $150 / 100$ & High \\
Subj17 & F & 45 & $140 / 80$ & High \\
Subj18 & F & 45 & $170 / 100$ & High \\
Subj19 & M & 20 & $110 / 70$ & Normal \\
Subj20 & M & 21 & $110 / 90$ & Normal \\
Subj21 & F & 20 & $110 / 80$ & Normal \\
Subj22 & F & 21 & $100 / 70$ & Normal \\
Subj23 & F & 22 & $100 / 60$ & Normal \\
Subj24 & M & 23 & $120 / 80$ & Normal \\
Subj25 & M & 21 & $120 / 80$ & Normal \\
\hline & & & &
\end{tabular}

especially in the emergency departments. The challenge can be potentially mitigated by using one of the modern smartwatches that are commercially available. But unfortunately, the collection of the raw PPG data from the sensor in those cases is not very straightforward. Given all these factors, we designed an embedded system (device) in order to acquire data. This device consists of a pulse sensor, connected to the Analog-to-Digital converter of an Arduino board. The sensor records PPG signals from the patients whose BP is measured alongside. Therefore, data collected from these experiments can be used to establish an effective connection between the morphological changes taking place in the PPG signals and its relation to one of the most important biomarkers that are used to assess the health of a human i.e., Blood pressure.

The data that has been used for our experiments have been collected at the Combined Military Hospital, Rawalpindi, Pakistan during routine clinical practice. The patients were paying a visit to the medical specialist before which, the $\mathrm{BP}$ readings of the patients were recorded. During the measurement of $\mathrm{BP}$, the device that we designed was used to collect the PPG signals from the fingertips of the patients. In total, we collected data from 25 patients out of which 14 patients had normal BP and 11 patients had abnormal BP. The names and identity of the patients are anonymized to protect the privacy of the patients. It is important to note that in all the data that has been collected, there were only two patients where the BP reading showed an abnormal value. This is a small number and any machine learning algorithm will not be able to perform classification with a satisfactory, statistically significant analysis. Given this, we aim to focus on a detection 
problem i.e., we aim to explore, provided a PPG signal, whether the patient has normal BP or abnormal BP.

It should be noted that we only have two cases of patients with low BP. If we go for a three-class problem, a reliable estimate of classification is not possible. Moreover, the application we are focused at relates to preventive healthcare which will not require precise values of BP. Once an alarm is raised for a patient, they can be advised for a thorough check-up at a medical center. Given this, we aim for a two-class classification problem for identifying patients with BP issues: Normal BP and Abnormal BP. Any subject having BP between 100/60 and 130/90 is taken as normal, with measurements outside of this range regarded as abnormal. It should be noted that the $\mathrm{BP}$ values are shown in Table 1 are only used for labeling the data. The classification is performed using PPG signals and the last column of Table 1 is used as ground truth for validation of the classification performance.

\section{Proposed Approach}

The algorithm for feature extraction consists of three stages: 1). Preprocessing, 2). Peak detection, 3). Feature extraction, and 4). Classification.

\subsection{Preprocessing}

There are several challenges that have to be addressed before the data can be presented for feature extraction. The most significant issue is that the sensor is placed at the fingertips and thus, a slight movement of the sensor from its position can be a significant source of the noise. This source of noise can corrupt the high-frequency content of the PPF signal. Additionally, there can be some missing samples in the data along with the presence of outliers (samples whose magnitudes exceed a certain defined range of permissible values) which can skew the results in an undesired manner.

In view of the above, the acquired signal is first treated for outlier removal. Here, outliers are defined as either missing data points or the data points which exhibit values that are greater than 20 times the median waveform height. After outlier removal, the signal is subjected to low pass Butterworth filtering [49]. This is because PPG is a low-frequency signal given that the maximum number of beats per minute for humans is limited to 200 beats per minutes. It should be noted that the application of this filter on the data will remove the noise that was potentially corrupting the high-frequency content in the signal. In view of this, the cutoff frequency of the Butterworth filter is kept at $7 \mathrm{~Hz}$ to ensure that we sample the PPG signal at the Nyquist rate. It is expected that the neighboring samples in the acquired signals should be highly correlated and therefore a moving average filter is applied for noise removal from the PPG signal.

\subsection{Peak detection}

After preprocessing, the filter is subjected to peak detection. The peak detection usually uses the zero derivative method which is very sensitive to noise in the real signals. To cater for this issue, we use the peak detection algorithm proposed by Billauer et al. [50]. This algorithm is based on a simple idea that a peak (maxima) is the highest point in a signal between the valleys (minima). All points lying around a peak should exhibit a lower amplitude. In this way, the algorithm considers a variable window where peaks are identified around which adjacent data points have a lower magnitude than a threshold. This peak can be fine-tuned so that the smaller peaks are discarded.

\subsection{Feature extraction}

The peak detection phase is followed by the feature extraction phase (Fig. 3). A PPG waveform along with its physiological labeling is shown in Fig. 4. In view of this, we have identified three important features which can be used for calculating the BP [16]. These features are, i). rising time, ii). falling time and iii). peak magnitude. The rising time is calculated in terms of the slope of the PPG signal in the cycle that is preceding the peak (positive slope) whereas the falling time is calculated in terms of the slope following the peak in PPG signals (negative slope). These features can be calculated for every cycle in the PPG signals. Finally, we obtain distinct vectors for each of the three features that have been calculated from the signal. Finally, we obtain a histogram for each feature individually and concatenate them forming our final feature vector that can be used to perform the subject classification task.

\subsection{Classification}

We have chosen different statistical classifiers in our work, their choice is made by their main archetypes as explained below:

Nearest Neighbor (NN): This classifier labels an unknown object with the label of the majority class of its ' $k$ ' neighbors in the feature space [51]. The distance between an object and its nearest neighbors is measured in the Euclidean sense.

Support Vector Machines (SVM): The SVM, originally proposed by Vapnik et al. [52] mainly consists of constructing an optimum hyperplane that maximizes the margin of separation between two different classes. This approach typically constructs the classification models which have excellent generalization ability thus making it a powerful tool in various applications. For our implementation, we have used SVM with the linear kernel for classification.

Decision Trees (DT): This classifier uses trees that can be modeled according to a set of decision rules [53]. The learning phase generates a binary tree structure that contains the rules that effectively predict the target variable. 


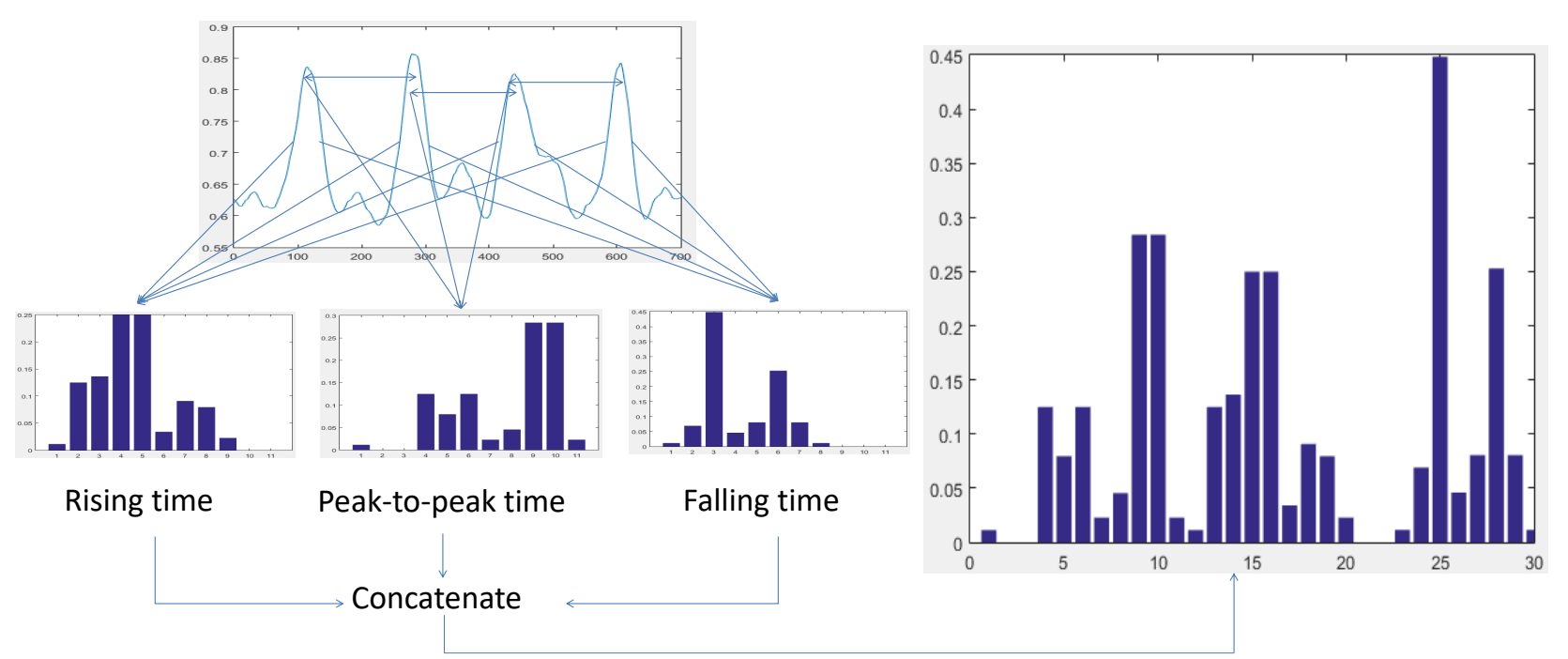

Figure 3: The features extracted from PPG signals i.e., the histograms of rising time, falling time and peak-to-peak time.

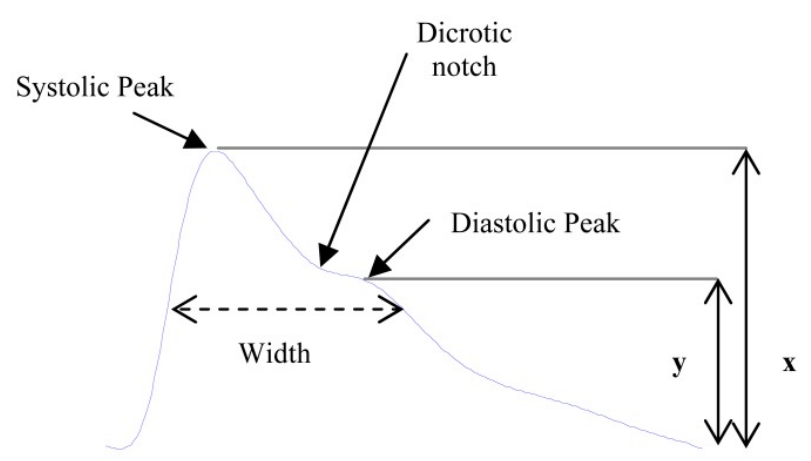

Figure 4: Typical waveform of PPG and labeling of its characteristic parameters.

Neural Networks: Neural networks are computational methods that are inspired by the biological neural systems that constitute the human brains [52]. The goal of this method is to emulate the human brain by making decisions followed by backpropagation (passing information as feedback) and adjust the network to reflect this information.

\subsection{Weighted Voting of classification}

The feature extraction module is followed by feature selection. In the proposed method, the feature extraction methodology yields a small number of features. This is because of the rising time, falling time and peak-to-leak times are histogrammed with a small number of bins. Although the number of features is already limited, the contribution of each feature towards classification is significantly different. This is evident from a statistical analysis of the histograms showing that the features with the highest significance are the peak-to-peak times, followed by the rising time, and then the falling time of the PPG signal. Given

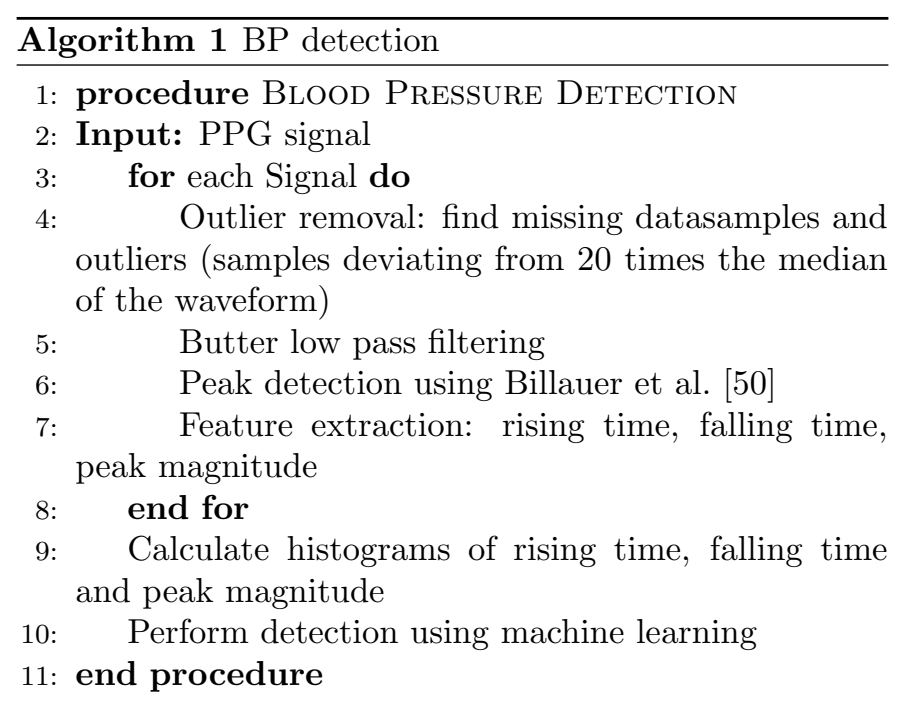

this, it is clear that the contribution of each feature towards classification is different. Consequently, we have to learn the weights corresponding to the contribution of these features. For simplicity, assuming that there exists an autoregressive (AR) model between the actual and predicted outcome, we know that

$$
X=w \cdot P
$$

where $w$ is a vector having the AR predictive coefficients. The weights can be calculated using the Least square sense as follows:

$$
w=\left(X^{T} X\right)^{-1} X^{T} P
$$

where $\left(X^{T} X\right)^{-1} X^{T}$ is the pseudo-inverse of the matrix $X$. Once the weights are learned using the training data, they are used for the testing data such that the outputs of classifiers using individual features are obtained followed 
by their corresponding weighting using the weights learned through the autoregressive (AR) models, giving the final output of the classifiers.

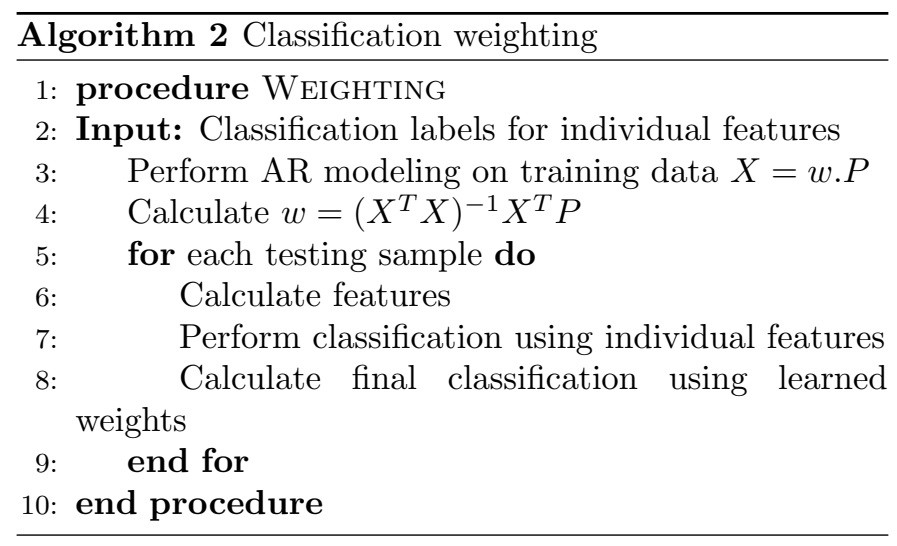

\section{Experimental setup}

One of the challenges that are faced in this study is the amount of dataset to validate the proposed method, which is limited. We have data available from 25 patients which is a relatively small number in order to reliably train the classifier. Therefore, it is not feasible to split the dataset into training and testing sets as this will not result in the performance evaluation that is statistically significant. To mitigate this problem, we opted to perform crossvalidation for training and testing. We have performed two types of cross-validation: 10-fold cross-validation (CV) and leave-one-out cross-validation (LOOCV). Although we are aware that LOOCV would result in overfitting, however, it is inevitable to use it since the size of the dataset is small. To further strengthen the experimental setup and validate the statistical significance of the experimental evaluation, we repeated the experiments 100 times using random configuration (selection) of the training and testing folds (for 10 fold CV). To this extent, we have used paired t-test to perform significance testing of the performance.

In our experiments, we have used four different classification algorithms to validate the performance: SVM, 1NN and DT. This choice is motivated by the fact that each one of them presents a distinct class of machine learning methods. As compared to the neural networks, all these methods belong to relatively simpler types of algorithms. SVMs (linear kernel) and 1NN are linear classifiers and using these methods, it is possible to assess the strength of the feature extraction methods. This is because by using a complex classifier such as neural networks, the load of better classification is shifted onto the classifiers and it is not possible to evaluate the strength of the features.

Most state-of-the-art methods used for calculation of BP use signals from two different modalities: PPG and ECG. The correlation between BP and PTT is a feature

\begin{tabular}{c|c|c|c|c}
\multicolumn{2}{c}{} & \multicolumn{2}{c}{ Predicted } & \multirow{2}{c}{ Total } \\
\cline { 2 - 4 } & normal & abnormal & \\
\cline { 2 - 4 } Actual & normal & $T P$ & $F N$ & $F N$ \\
\cline { 2 - 4 } & abnormal & $F P$ & $T N$ & $N N$ \\
\cline { 2 - 4 } & Total & $T P+F P$ & $F N+T N$ & $N$
\end{tabular}

Table 2: Confusion Matrix.

that is utilized to calculate the BP which is measured using both the PPG and ECG signals. Given that our objective in this paper is asses a direct correlation between BP and PPG signals, we extract the features directly from the PPG signals and classify the signals as being acquired from a subject having normal or abnormal BP. So we do not perform any comparison with any other feature extraction methods. We do understand that we are not measuring the exact reading of the BP, but the aim of this study is to devise a mechanism using which an alarm can be generated so that the users can seek medical attention. Consequently, the scope of the problem involves the identification of abnormal blood pressure and is thus a two class problem.

As an extension to the existing methods, we have also performed weighting of classifier outputs using auto-regressive modeling. To achieve this, we perform 10-fold cross-validation. The weights, $w$ are learned using the training sets and later the classification outputs using individual features is obtained followed by weighting using the learned weights. We have not used LOOCV on this experiment as this could lead to over-fitting of the learned weights.

\subsection{Evaluation Metric}

The proposed approach is evaluated using standard machine learning and information retrieval metrics, namely, the true positive rate (TPR), the false positive rate (FPR), the precision, the recall, and the F-score. The TPR represents the ratio of objects classified to have high blood pressure to the total number of objects who actually have high blood pressure in the dataset. The FPR represents the ratio of objects with normal blood pressure classified as abnormal blood pressure. The F-Score is the harmonic mean of precision and recall as given in an Equation 5.

$$
\begin{gathered}
\text { Recall }=\frac{T P}{T P+F N} \\
\text { Precision }=\frac{T P}{T P+F P} \\
F-\text { Score }=2 * \frac{\text { Precision } * \text { Recall }}{\text { Precision }+ \text { Recall }}
\end{gathered}
$$

\section{Performance Analysis}

In this section, we present performance results for different machine learning methods. 
Table 3: p-values obtained for different types of features for discriminating between normal and abnormal BP

\begin{tabular}{cc}
\hline Features & p-values \\
\hline Rising time & 0.0043 \\
Peak-to-peak time & $6.53 \mathrm{e}-04$ \\
Falling time & 0.83 \\
\hline
\end{tabular}

\subsection{Basic Classification Results}

An immediate conclusion from the experiments is that the proposed methodology can be used for the detection of BP abnormalities with high accuracy (Table 4 and 5). Experiments show that all classification methods perform reasonably well with neural networks demonstrating best overall results. The good performance of SVM with a linear kernel indicates that the features we have obtained are linearly separable. Therefore, they are discriminative and can be linearly mapped towards the detection of BP problems. The results are consistent irrespective of the test bed used to carry out the experiments (10-fold cross-validation and LOOCV). In addition to the classification accuracy, we have also calculated the running time of the proposed algorithm. To do this, we record a small time window of a signal ( $5 \mathrm{~s}$ window) and calculate the temporal features from the signal in Matlab running on a computer with Core i5 processor and 4GB RAM. For one window, the feature histogram was calculated within $0.21 \mathrm{~s}$. This indicates that the algorithm is fast and has potentials for real-time monitoring of the $\mathrm{BP}$ anomalies.

\subsection{Strength of individual features}

We have used histograms of three distinct features from the PPG signal: 1) rising time, 2) falling time and 3) peakto-peak distance. In order to assess the behavior of these features towards discriminating normal BP from the abnormal, we have performed significance testing on each of these features i.e. adopting features for the positive and negative classes and performed a t-test on them.

Our experiments show that falling times for the positive and negative class are not significantly different and thus the relevant features are not a powerful indicator of discrimination between the two classes. In contrast, the rising time has a higher significance $(p=0.0043)$ whereas the peak-to-peak time has the highest significance $(\mathrm{p}=6.53 \mathrm{e}-$ 04) indicating that these features have the highest discrimination capability towards identifying abnormal BP (Table 6). A visual representation of the significance of these features (Figure 5) shows that the peak-to-peak times of these features vary significantly as compared to the other features that have been considered in this paper. An overlap in the normal and abnormal BP falling times exists as shown in figure 5 reveals that it has the least discrimination capability.

Although these results are encouraging, it is possible to expand the feature set to obtain better classification results if spectral features are employed. This is because of the fact that the rising time, falling time and interpeak distance are also related to the spectral content of the signal which can be effectively extracted to give more relevant features for the classification of the signals.

\subsection{Weighted voting}

The experimental setup for the weighted voting is different from the setup that was used for the evaluation of classification results. The aim of this experiment was to assess the individual contribution of each feature set towards classification. Out of a total of 24 samples, 10 samples were left out to perform the testing of the weighted voting. The remaining 14 samples were used for learning the weights of individual features using LOOCV. The classification was performed using each feature set separately and the predicted outputs were obtained using only one classification method i.e., KNN classifier. Our choice is motivated by the fact that 1-nearest neighbor is the simplest classifier that uses a linear measure (Euclidean distance) in the feature space to measure the similarity of the features and thus, it truly derive the essence of the strength of the individual features towards the subject classification problem.

The experiment was repeated 10 times using random folds in each experiment. The predicted labels were obtained and compared with the actual labels and the weight vector was obtained with three coefficients, one belonging to each feature set. The experiments were performed on the 10 samples, which were kept hidden from the entire series of experiments. Now, to calculate the impact of these learned weights on classification, each feature set was used separately to obtain its respective class labels and the weights were applied to these labels to find the final prediction of the class label. The 14 samples that were used for learning the weights were reused in this experiment as training data whereas testing was performed on the 10 samples that were completely blind to the training phase. The 10 samples were selected such that there were 5 samples from each class (normal vs. abnormal BP). Given this, the TP rate can be directly used for evaluation of the classifier performance and no other measure is required since the testing sets are equal in size.

Our experiments show that irrespective of the classification methodology used, the results using weighted voted have been improved. The best results are obtained using 10-fold cross-validation with an overall accuracy of about $98 \%$. Although we know that results with better statistical significance could be obtained if more data was available, this experiment has validated our hypothesis that the contribution of peak-to-peak distance and rising time is the most significant in the identification of $\mathrm{BP}$ in the patients.

Finally, we have compared the proposed feature extraction methodology with two other types of features (Table 9): spectral features (including spectral centroid and spectral variance calculated from the power spectral density of the PPG signal) and the wavelet-based feature (mean 
Table 4: Overall classification results for the detection of BP using different machine learning methods with 10-fold cross validation.'*, indicates statistically significant results with respect to the nearest neighbor.

\begin{tabular}{ccccccc}
\hline Methods & TP Rate & FP Rate & Precision & Recall & F-measure & ROC area \\
\hline Nearest Neighbor & 0.90 & 0.18 & 0.91 & 0.98 & 0.94 & 0.83 \\
Support Vector Machine & 0.95 & 0.09 & 0.96 & 0.99 & 0.97 & $0.95^{*}$ \\
Decision Trees & $0.94^{*}$ & $0.11^{*}$ & $0.95^{*}$ & 0.99 & $0.97^{*}$ & $0.94^{*}$ \\
Neural Networks & $\mathbf{0 . 9 5 *}$ & $\mathbf{0 . 0 9}$ & $\mathbf{0 . 9 6}$ & $\mathbf{0 . 9 9 *}$ & $\mathbf{0 . 9 7}^{*}$ & $\mathbf{0 . 9 9}^{*}$ \\
\hline
\end{tabular}

Table 5: Overall classification results for the detection of BP using different machine learning methods with Leave-one-out cross-validation. ${ }^{*}$ ' indicates statistically significant results with respect to the nearest neighbor.

\begin{tabular}{ccccccc}
\hline Methods & TP Rate & FP Rate & Precision & Recall & F-measure & ROC area \\
\hline Nearest Neighbor & 0.91 & 0.15 & 0.90 & 0.97 & 0.94 & 0.85 \\
Support Vector Machine & $0.95^{*}$ & 0.10 & $0.93^{*}$ & 0.98 & $0.97^{*}$ & $0.96^{*}$ \\
Decision Trees & 0.93 & 0.13 & 0.94 & 0.99 & $0.97^{*}$ & $0.97^{*}$ \\
Neural Networks & $\mathbf{0 . 9 6}^{*}$ & $\mathbf{0 . 0 8 *}$ & $\mathbf{0 . 9 5}^{*}$ & $\mathbf{0 . 9 9}^{*}$ & $\mathbf{0 . 9 6}^{*}$ & $\mathbf{0 . 9 9}^{*}$ \\
\hline
\end{tabular}

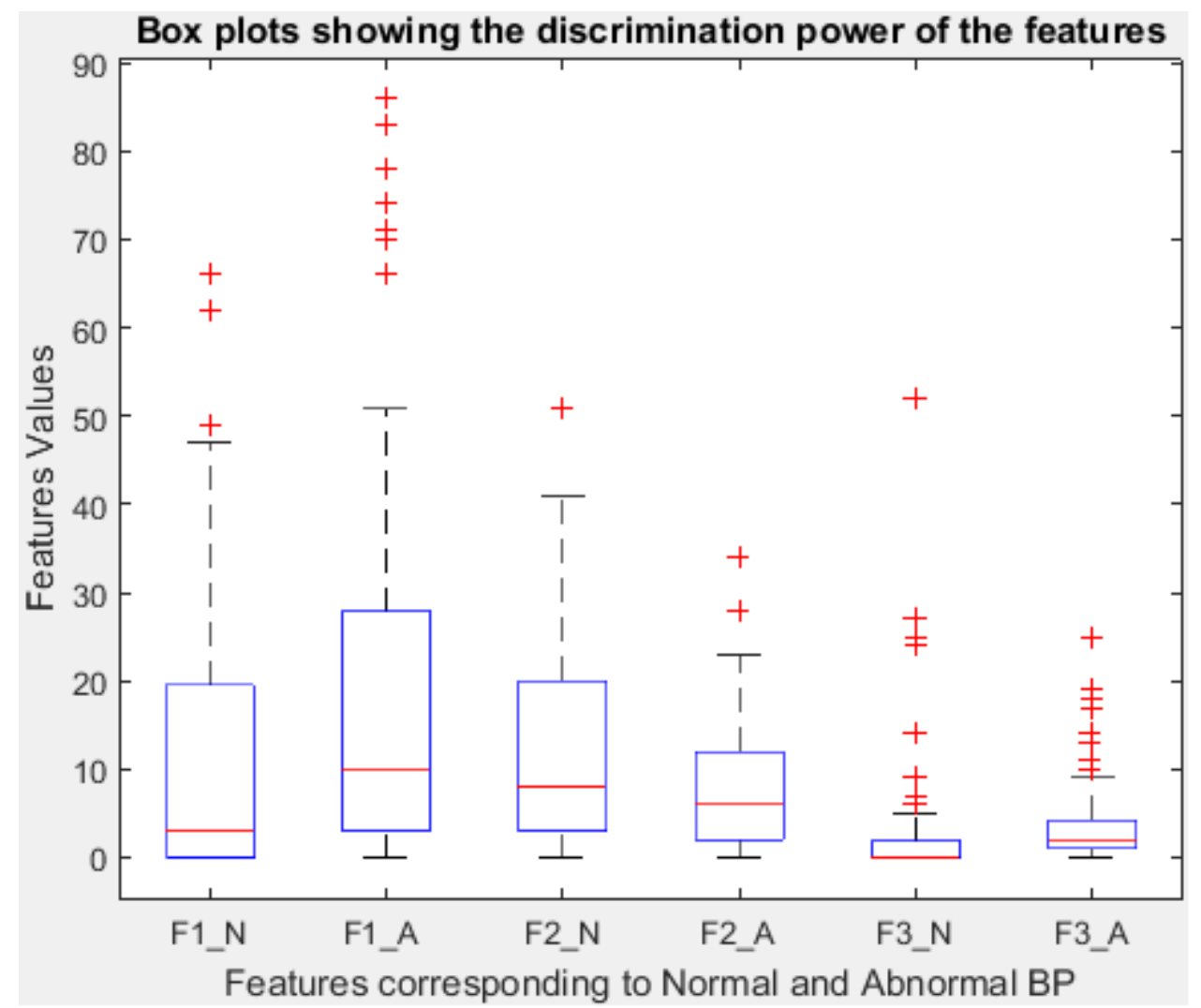

Figure 5: The boxplots of different types of features which are used for feature extraction (F1_N: Rising time for Normal BP; F1_A: Rising time for abnormal BP; F2_N: Peak-to-peak time for normal BP; F2_A: Peak-to-peak time for abnormal BP; F3_N: Falling time for normal BP; F3_A: Falling time for abnormal BP).

and variance values of the decomposed signals). The experiments were performed using 10 -fold cross-validation with the experiments reproduced using 10 random runs (folds) and classification is done using SVMs. The experiments show that the proposed feature set outperforms the other methods that were considered in the paper, hinting at the powerful nature of the features which are physio- logically motivated.

\section{Discussion}

This study is aimed at investigating the correlation between PPG signals and their ability to detect BP abnormalities. Literature shows that the use of ECG and PPG 
Table 6: Classification using individual feature set

\begin{tabular}{cc}
\hline Features & TP rate $( \pm \sigma)$ \\
\hline Rising time & $92.86 \pm 0.03$ \\
Peak-to-peak time & $92.86 \pm 0.03$ \\
Falling time & $91.43 \pm 0.02$ \\
\hline
\end{tabular}

together can help calculate BP directly, however, synchronization of both signals simultaneously is not an easy task. Additionally, the calculation of BP requires placing multiple sensors on the body which is non-trivial. Consequently, more sensors we have, more challenging it becomes to calculate the BP. Within this context, our aim in this paper is to use only PPG signals in the detection of abnormal BP. It should be noted that our aim is not to eventually design a medical grade device that can precisely calculate the BP but to design a system that can be used to raise the relevant alarms if an individual has abnormal BP. We exploit the potential of the PPG signals in predicting BP based on the rising time, falling time and inter-peak intervals of the PPG waveform. The signal is first filtered using Butterworth and averaging filters for the noise removal followed by the detection of the peaks. The temporal features from the cleansed signal are then extracted followed by the classification of the signal into two classes abnormal and normal BP.

We have used two distinct variants for the classification task. In the first one, we used 10-fold cross-validation (CV) and leave-one-out CV (LOOCV). In these experiments, the proposed feature set performed very well in identifying abnormal BP. Given that we mainly focused on preventive healthcare, the aim of our classification task is to detect if a patient has normal or abnormal BP (two-class problem). The best performance was achieved using neural networks. The other simple classifiers also performed well indicating that the feature set is powerful indeed and inculcates strong potential towards the identification of BP. Additionally, a deeper study performed on the features indicates that their significance towards classification exhibits variations i.e. peak-to-peak distances have a stronger statistical significance towards the identification of BP. This set us to perform our second experiment in which we assign weights to the classification results obtained using different feature sets and then applying those weights to an unseen data that is kept completely hidden from the training phase or learning of weights. The unseen data was split into training and testing sets to make the evaluation easier. Our experiments confirmed that the assignment of these weights significantly improves the classification results producing an overall accuracy of about $98 \%$. Conclusively, our experiments show that the proposed method can effectively detect blood pressure abnormalities with good accuracy, although further robust statistical validation on more data is required.

In the future, we aim to expand the feature set to fur- ther improve the classification results. Additionally, we would like to expand the dataset to include further cases of normal and abnormal BP. Further, we would like to perform data collection with more classes such as very low, low, normal, high and very high BP. This could further increase the impact of the work and make the research more applicable for preventive healthcare.

\section{Acknowledgements}

Imran's work is supported by the Deanship of Scientific Research, King Saud University through research group project number RG-1435-051.

\section{References}

[1] P. Lamkin. (2018) Wearable tech market to double by 2021. [Online]. Available: https://goo.gl/MvspV9

[2] Y. Lin, C. Hung, H. Tsai, and K. Huang, "Evaluation of blood pressure by analyzing the fluctuation frequency of oxygen saturation in palmar digital vein," in 2017 IEEE International Instrumentation and Measurement Technology Conference (I2MTC), May 2017, pp. 1-5.

[3] T. Sirkis, Y. Beiderman, S. Agdarov, Y. Beiderman, and Z. Zalevsky, "Monitoring blood vital bio signs using secondary speckle patterns," Opt. Express, vol. 24, no. 24, pp. 27899-27909, Nov 2016. [Online]. Available: http://www.opticsexpress.org/abstract.cfm?URI=oe-2424-27899

[4] V. K. van Wijnen, C. Finucane, M. P. M. Harms, H. Nolan, R. L. Freeman, B. E. Westerhof, R. A. Kenny, J. C. ter Maaten, and W. Wieling, "Noninvasive beat-to-beat finger arterial pressure monitoring during orthostasis: a comprehensive review of normal and abnormal responses at different ages," Journal of Internal Medicine, vol. 282, no. 6, pp. 468-483. [Online]. Available: https://onlinelibrary.wiley.com/doi/abs/10.1111/joim.12636

[5] A. P. Avolio, M. Butlin, and A. Walsh, "Arterial blood pressure measurement and pulse wave analysistheir role in enhancing cardiovascular assessment," Physiological Measurement, vol. 31, no. 1, p. R1, 2010. [Online]. Available: http://stacks.iop.org/0967-3334/31/i=1/a=R01

[6] T. Khamdaeng, J. Luo, J. Vappou, P. Terdtoon, and E. Konofagou, "Arterial stiffness identification of the human carotid artery using the stressstrain relationship in vivo," $U l$ trasonics, vol. 52, no. 3, pp. $402-411,2012$.

[7] J. Havlik, Z. Martinovská, J. Dvorák, and L. Lhotská, "Detection of artifacts in oscillometric pulsations signals," in Proceedings of 2014 IEEE-EMBS International Conference on Biomedical and Health Informatics (BHI). IEEE, 2014, pp. 709-711.

[8] M. F. ORourke and A. Adji, "Noninvasive generation of aortic pressure from radial pressure waveform by applanation tonometry, brachial cuff calibration, and generalized transfer function," American journal of hypertension, vol. 27, no. 2, pp. 143-145, 2013.

[9] C.-S. Kim, A. M. Carek, R. Mukkamala, O. T. Inan, and J.O. Hahn, "Ballistocardiogram as proximal timing reference for pulse transit time measurement: Potential for cuffless blood pressure monitoring," IEEE Transactions on Biomedical Engineering, vol. 62, no. 11, pp. 2657-2664, 2015.

[10] Y. Zheng, B. P. Yan, Y. Zhang, C. Yu, and C. C. Poon, "Wearable cuff-less ptt-based system for overnight blood pressure monitoring," in Proceedings of 2013 35th IEEE Annual International Conference Engineering in Medicine and Biology Society (EMBC),. IEEE, 2013, pp. 6103-6106. 
Table 7: Overall classification results for the detection of BP using different machine learning methods with 10-fold cross-validation. '*, indicates statistically significant results with respect to the nearest neighbor.

\begin{tabular}{ccccccc}
\hline Methods & TP Rate & FP Rate & Precision & Recall & F-measure & ROC area \\
\hline Nearest Neighbor & 0.92 & 0.10 & 0.93 & 0.92 & 0.92 & 0.88 \\
Support Vector Machine & $\mathbf{0 . 9 8}^{*}$ & $\mathbf{0 . 0 5 *}$ & $\mathbf{0 . 9 6}$ & $\mathbf{0 . 9 6}$ & $\mathbf{0 . 9 6}^{*}$ & $\mathbf{0 . 9 6}^{*}$ \\
Decision Trees & 0.95 & 0.15 & 0.90 & 0.88 & 0.88 & 0.78 \\
Neural Networks & $0.96^{*}$ & $0.05^{*}$ & $0.96^{*}$ & $0.96^{*}$ & $0.96^{*}$ & $0.99^{*}$ \\
\hline
\end{tabular}

Table 8: Overall classification results for the detection of BP using different machine learning methods with Leave-one-out cross-validation. '*' indicates statistically significant results with respect to the nearest neighbor.

\begin{tabular}{ccccccc}
\hline Methods & TP Rate & FP Rate & Precision & Recall & F-measure & ROC area \\
\hline Nearest Neighbor & 0.92 & 0.10 & 0.93 & 0.92 & 0.92 & 0.82 \\
Support Vector Machine & $0.96^{*}$ & 0.05 & 0.96 & $0.96^{*}$ & $0.96^{*}$ & $0.96^{*}$ \\
Decision Trees & 0.96 & 0.05 & 0.96 & 0.96 & 0.96 & 0.91 \\
Neural Networks & $\mathbf{0 . 9 6}^{*}$ & $\mathbf{0 . 0 5}$ & $\mathbf{0 . 9 6}^{*}$ & $\mathbf{0 . 9 6}$ & $\mathbf{0 . 9 6}^{*}$ & $\mathbf{0 . 9 9}^{*}$ \\
\hline
\end{tabular}

Table 9: Comparison with other feature sets. '*' indicates statistically significant results with respect to wavelet-based features.

\begin{tabular}{cc}
\hline Features & TP rate $( \pm \sigma)$ \\
\hline Proposed features & $\mathbf{0 . 9 8} * \mathbf{0 . 0 1}$ \\
Spectral features & $0.92 \pm 0.02$ \\
Wavelet-based features & $0.94 \pm 0.03$ \\
\hline
\end{tabular}

[11] X.-R. Ding, Y.-T. Zhang, J. Liu, W.-X. Dai, and H. K. Tsang, "Continuous cuffless blood pressure estimation using pulse transit time and photoplethysmogram intensity ratio," IEEE Transactions on Biomedical Engineering, vol. 63, no. 5, pp. 964-972, 2016.

[12] R. Mukkamala, J.-O. Hahn, O. T. Inan, L. K. Mestha, C.-S. Kim, H. Töreyin, and S. Kyal, "Toward ubiquitous blood pressure monitoring via pulse transit time: theory and practice," IEEE Transactions on Biomedical Engineering, vol. 62, no. 8, pp. 1879-1901, 2015.

[13] TURNBULL, "Effects of different regimens to lower blood pressure on major cardiovascular events in older and younger people: meta-analysis of randomised trials," Biomedical Journal, vol. 336, pp. 1121-1123, 2008 .

[14] J. Allen, "Photoplethysmography and its application in clinical physiological measurement," vol. 28, 012007.

[15] A. D. Choudhury, R. Banerjee, A. Sinha, and S. Kundu, "Estimating blood pressure using windkessel model on photoplethysmogram," in Proceedings of 2014 36th Annual International Conference of the IEEE Engineering in Medicine and Biology Society (EMBC). IEEE, 2014, pp. 4567-4570.

[16] A. Visvanathan, A. Sinha, and A. Pal, "Estimation of blood pressure le vels from reflective photoplethysmograph using smart phones," in Proceedings of 2013 IEEE 13th International Conference on Bioinformatics and Bioengineering (BIBE),. IEEE, 2013, pp. 1-5.

[17] T. Aoyagi, "Pulse oximetry: its invention, theory, and future," Journal of Anesthesia, vol. 17, pp. 259-266, 2003.

[18] J. L. Moraes, M. X. Rocha, G. G. Vasconcelos, J. E. V. Filho, V. H. C. de Albuquerque, and A. R. Alexandria, "Advances in photopletysmography signal analysis for biomedical applications," vol. 18 , no. 6 , p. 1894, 2018.

[19] A. T. Reisner, P. A. Shaltis, D. Mccombie, and H. H. Asada, "Utility of the photoplethysmogram in circulatory monitoring." Anesthesiology, vol. 108 5, pp. 950-8, 2008.
[20] T. Tamura, Y. Maeda, M. Sekine, and M. Yoshida, "Wearable photoplethysmographic sensorspast and present," Electronics, vol. 3, no. 2, pp. 282-302, 2014.

[21] S. Fleming and L. Tarassenko, "A comparison of signal processing techniques for the extraction of breathing rate from the photoplethysmogram," International Journal of Biological and Medical Sciences.

[22] A. Fusco, D. Locatelli, F. Onorati, G. C. Durelli, and M. D. Santambrogio, "On how to extract breathing rate from ppg signal using wearable devices," in 2015 IEEE Biomedical Circuits and Systems Conference (BioCAS), Oct 2015, pp. 1-4.

[23] P. A. Leonard, J. G. Douglas, N. R. Grubb, D. Clifton, P. S. Addison, and J. N. Watson, "A fully automated algorithm for the determination of respiratory rate from the photoplethysmogram," Journal of Clinical Monitoring and Computing, vol. 20, no. 1, pp. 33-36, Feb 2006.

[24] P. Charlton, T. Bonnici, L. Tarassenko, D. Clifton, R. Beale, and P. J Watkinson, "An assessment of algorithms to estimate respiratory rate from the electrocardiogram and photoplethysmogram," Physiological measurement, vol. 37, pp. 610-626, 03 2016.

[25] M. Elgendi, "On the analysis of fingertip photoplethysmogram signals," Current cardiology reviews, vol. 8, pp. 14-25, 022012.

[26] M. Sharma, K. Barbosa, V. Ho, D. Griggs, T. Ghirmai, S. K. Krishnan, T. K. Hsiai, J.-C. Chiao, and H. Cao, "Cuff-less and continuous blood pressure monitoring: A methodological review," Technologies, vol. 5, no. 2, 2017. [Online]. Available: http://www.mdpi.com/2227-7080/5/2/21

[27] B. Y. Su, M. Enayati, K. C. Ho, M. Skubic, L. Despins, J. M. Keller, M. Popescu, G. Guidoboni, and M. Rantz, "Monitoring the relative blood pressure using a hydraulic bed sensor system," IEEE Transactions on Biomedical Engineering, pp. 1-1, 2018.

[28] P. A. Shaltis, A. Reisner, and H. H. Asada, "Wearable, cuffless ppg-based blood pressure monitor with novel height sensor," in Proceedings of 2006 IEEE International Conference in Medicine and Biology Society, Aug 2006, pp. 908-911.

[29] S. S. Thomas, V. Nathan, C. Zong, E. Akinbola, A. L. P. Aroul, L. Philipose, K. Soundarapandian, X. Shi, and R. Jafari, "Biowatch a wrist watch based signal acquisition system for physiological signals including blood pressure," in Proceedings of 2014 36th Annual International IEEE Conference of the Engineering in Medicine and Biology Society, Aug 2014, pp. 2286-2289.

[30] Y. Yoon, J. H. Cho, and G. Yoon, "Non-constrained blood pressure monitoring using ecg and ppg for personal healthcare," Journal of Medical Systems, vol. 33, pp. 261-266, 2008.

[31] L. C. L. Landicho, I. R. Magbalon, and C. P. S. Reyes, "A 
wireless electrocardiogragphy in superintending cardiac rate extremes with global positioning capability to remotely localize specimen," in 2015 IEEE International Conference on Control System, Computing and Engineering (ICCSCE), Nov 2015, pp. 141-145.

[32] X. Zhang, Y. Lyu, X. Luo, J. Zhang, C. Yu, H. Yin, and Y. Shi, "Touch sense: Touch screen based mental stress sense," Proc. ACM Interact. Mob. Wearable Ubiquitous Technol., vol. 2, no. 2, pp. 87:1-87:18, Jul. 2018. [Online]. Available: http://doi.acm.org/10.1145/3214290

[33] C. Choi, B. Ko, J. Lee, S. K. Yoon, U. Kwon, S. J. Kim, and Y. Kim, "Ppg pulse direction determination algorithm for ppg waveform inversion by wrist rotation," in 2017 39th Annual International Conference of the IEEE Engineering in Medicine and Biology Society (EMBC), July 2017, pp. 4090-4093.

[34] C. Choi, B.-H. Ko, J. Lee, S. K. Yoon, U. Kwon, S. J. Kim, and Y. Kim, "Ppg pulse direction determination algorithm for ppg waveform inversion by wrist rotation," in 201739 th Annual International Conference of the IEEE Engineering in Medicine and Biology Society (EMBC). IEEE, 2017, pp. 4090-4093.

[35] W. B. Gu, C. C. Y. Poon, H. K. Leung, M. Y. Sy, M. Y. M. Wong, and Y. T. Zhang, "A novel method for the contactless and continuous measurement of arterial blood pressure on a sleeping bed," in Proceedings of 2009 Annual International Conference of the IEEE Engineering in Medicine and Biology Society, Sept 2009, pp. 6084-6086.

[36] Y. Kurylyak, F. Lamonaca, and D. Grimaldi, "A neural network-based method for continuous blood pressure estimation from a ppg signal," in Proceedings of 2013 IEEE International Instrumentation and Measurement Technology Conference (I2MTC), May 2013, pp. 280-283.

[37] A. F. Hussein, A. k. N, M. Burbano-Fernandez, G. RamrezGonzlez, E. Abdulhay, and V. H. C. D. Albuquerque, "An automated remote cloud-based heart rate variability monitoring system," IEEE Access, vol. 6, pp. 77 055-77 064, 2018.

[38] J. J. P. C. Rodrigues, D. B. D. R. Segundo, H. A. Junqueira, M. H. Sabino, R. M. Prince, J. Al-Muhtadi, and V. H. C. D. Albuquerque, "Enabling technologies for the internet of health things," IEEE Access, vol. 6, pp. 13129-13141, 2018.

[39] M. M. E. Mahmoud, J. J. P. C. Rodrigues, S. H. Ahmed, S. C. Shah, J. F. Al-Muhtadi, V. V. Korotaev, and V. H. C. D. Albuquerque, "Enabling technologies on cloud of things for smart healthcare," IEEE Access, vol. 6, pp. 31950-31 967, 2018.

[40] J. Dey, A. Gaurav, and V. N. Tiwari, "Instabp: Cuff-less blood pressure monitoring on smartphone using single ppg sensor," in Proceedings of 2018 40th Annual International Conference of the IEEE Engineering in Medicine and Biology Society (EMBC). IEEE, 2018, pp. 5002-5005.

[41] S. S. Mousavi, M. Firouzmand, M. Charmi, M. Hemmati, M. Moghadam, and Y. Ghorbani, "Blood pressure estimation from appropriate and inappropriate ppg signals using a whole-based method," Biomedical Signal Processing and Control, vol. 47, pp. 196-206, 2019.

[42] G. Kalantar, S. K. Mukhopadhyay, F. Marefat, P. Mohseni, and A. Mohammadi, "Wake-bpat: Wavelet-based adaptive kalman filtering for blood pressure estimation via fusion of pulse arrival times," in Proceedings of 2018 IEEE International Conference on Acoustics, Speech and Signal Processing (ICASSP). IEEE, 2018, pp. 945-949.

[43] R. A. FISHER, "The use of multiple measurements in taxonomic problems," Annals of Eugenics, vol. 7, no. 2, pp. 179188.

[44] K. Coussement and K. W. D. Bock, "Customer churn prediction in the online gambling industry: The beneficial effect of ensemble learning," Journal of Business Research, vol. 66, no. 9, pp. 1629 - 1636, 2013, advancing Research Methods in Marketing.

[45] J. Arshad, M. M. Abdellatif, M. M. Khan, and M. A. Azad, "A novel framework for collaborative intrusion detection for $\mathrm{m} 2 \mathrm{~m}$ networks," in 2018 9th International Conference on Information and Communication Systems (ICICS), April 2018, pp. 1217
[46] A. Junaid, A. Muhammad Ajmal, A. Mohammad Mahmoud, U. R. Muhammad Habib, and K. Salah, "Colide: a collaborative intrusion detection framework for internet of things," in IET Networks, in press, 2018.

[47] M. L. Metersky, C. R. Frei, and E. M. Mortensen, "Predictors of pseudomonas and methicillin-resistant staphylococcus aureus in hospitalized patients with healthcare-associated pneumonia," Respirology, vol. 21, no. 1, pp. 157-163.

[48] M. A. Azad, J. Arshad, and F. Riaz, "SOCIO-LENS: spotting unsolicited caller through network analysis," in Guide to Vulnerability Analysis for Computer Networks and Systems - An Artificial Intelligence Approach, 2018, pp. 235-258.

[49] A. Widmann, E. Schröger, and B. Maess, "Digital filter design for electrophysiological data-a practical approach," Journal of neuroscience methods, vol. 250, pp. 34-46, 2015.

[50] E. Billauer, "Peak detection (peakdet)," 2012. [Online]. Available: http://www.billauer.co.il/peakdet.html

[51] C. M. Bishop, Pattern recognition and machine learning. springer, 2006.

[52] V. Vapnik, The nature of statistical learning theory. Springer science \& business media, 2013.

[53] L. Rokach and O. Maimon, Data mining with decision trees: theory and applications. World scientific, 2014. 